\title{
Documentos
}

\section{ATRAPAMIENTO DEL NERVIO PUDENDO: UN SÍNDROME POR CONOCER}

\author{
Rodrigo Lema C. ${ }^{1}$, Paolo Ricci A. ${ }^{1}$ \\ ${ }^{1}$ Unidad de Ginecología, Departamento de Ginecología y Obstetricia, Clínica Las Condes.
}

\begin{abstract}
RESUMEN
El atrapamiento del nervio pudendo comprende un síndrome descrito por primera vez en 1987. Entre los múltiples motivos de consulta se encuentra principalmente el dolor perineal y/o pelviano, lo que puede asociarse a disfunción urinaria, anal e incluso sexual. Las tres ramas terminales poseen en diferente proporción fibras motoras, sensitivas y autonómicas. Por ello su atrapamiento puede causar signos y síntomas de expresión en cualquiera de los tres ámbitos. En total se estima que el 30\% es autonómico y el $70 \%$ es somático ( $50 \%$ sensitivo y $20 \%$ motor). El dolor es la causa más común de consulta. Clásicamente se describe como perineal, que se agrava al sentarse, disminuye o desaparece al estar de pie, habitualmente ausente al acostarse, y no compromete el sueño. Para su diagnóstico se utiliza la certificación de 2 criterios mayores o 1 criterio mayor asociado a 2 criterios menores. El mejor esquema de tratamiento es secuencial y comprende las siguientes etapas: etapa 1 o de autocuidado, etapa 2 o de inyecciones perineurales y etapa 3 o cirugía de descompresión del nervio pudendo.
\end{abstract}

\section{PALABRAS CLAVES: Compresión del nervio pudendo, dolor perineal}

\section{SUMMARY}

The pudendal nerve entrapment syndrome was first described in 1987. The main complaint for consultation is pain, mainly located at the perineal and/or pelvic area; which can be associated to urinary, anal and even sexual dysfunction. The three main pudendal terminal branches contain motor, sensitive and autonomic fibers; this explains the diversity of signs and symptoms. $70 \%$ of nervous fibers are somatic (sensitive $50 \%$ and $20 \%$ motor), and the rest autonomic (30\%). The classic pain is described as perineal, worsens when seating, diminishes or disappears when standing, usually absent when lying down and it does not interfere with night rest. The diagnosis can be entertained when two major criteria or one major and two minor are present simultaneously according the punctuation score mentioned. The treatment should always be sequential and includes three main steps. The stage 1 is autoprotection (perineal, avoidance of sitting, climbing, etc), stage 2 is based in pudendal nerve perineural injections (anesthetics and corticosteroids) and stage 3 implies decompression surgery of the pudendal nerve.

\section{KEY WORDS: Pudendal nerve compression, perineal pain}

\section{INTRODUCCIÓN}

El atrapamiento del nervio pudendo (NP) comprende un síndrome descrito por primera vez en 1987 por Amarenco y cols (1). Con el pasar de los años se ha estudiado, relacionándolo con múltiples signos y síntomas, permitiendo una mayor comprensión y otorgando un tratamiento adecuado según la etiología en cada caso (2-4).

Entre los múltiples motivos de consulta se en- 
cuentra principalmente el dolor perineal y/o pelviano, lo que puede asociarse a disfunción urinaria, anal e incluso sexual. Por estos motivos se trata de un tema que necesariamente debe conocer el ginecólogo actual. Son síntomas expresados con una larga data y de tal severidad, que incluso se ha descrito que ha llevado a algunos pacientes a considerar el suicidio. Generalmente se trata de pacientes que deambulan consultando a múltiples médicos, al no encontrar respuesta y tratamiento adecuado para estas dolencias, siendo, no pocas veces catalogadas como pacientes "histéricas", o con alguna posible afección psicológica, al reiterar síntomas que pueden ser abigarrados, extraños 0 sin relación alguna entre sí, en los cuales no es fácil sospechar una etiología común. Sin duda se trata de síntomas y signos muy amplios, y con una gran variabilidad individual, lo que hace más difícil el diagnóstico.

Si buscamos alguna analogía, tal vez lo más cercano es el síndrome del túnel carpiano, aunque como veremos más adelante en el caso del síndrome de atrapamiento del nervio pudendo (SANP), tema de más reciente conocimiento, puede ser más complejo aún, al dar síntomas y signos en un sector anatómico tan amplio como es la pelvis.

De todo lo anterior podemos inferir con gran propiedad que tanto la incidencia como la prevalencia se encuentran absolutamente subestimadas. En Europa y Estados Unidos se calcula que 1 de cada 7 mujeres experimentará esta patología en forma transitoria o crónica, sin predilección por la edad.

A continuación, desarrollamos el tema, entregando las bases para su mejor comprensión, intentando contribuir a disminuir el subdiagnóstico que hemos observado en nuestra experiencia clínica.

\section{ANATOMÍA DEL NERVIO PUDENDO}

Es una anatomía compleja, sin embargo necesaria de entender para explicar la patología que su atrapamiento provoca.

EI NP se origina de los segmentos medulares sacros: S2, S3 y S4. Presenta tres zonas:

A. Primer segmento, comprendido desde su origen hasta la región presacra. No recibe contribución de S1 y S5.

B. Segundo segmento, que corresponde al canal infrapiriforme. Inmediatamente después de su comienzo, el NP penetra la región glútea bajo el músculo piriforme y cruza alrededor del extremo final del ligamento sacroespinoso. Aquí entra en contacto con el ligamento sacrotuberoso. Esta relación anatómica reviste una gran importancia, ya que en esta ubicación los ligamentos se comportan como una verdadera pinza, que puede comprimir al NP, causando su "atrapamiento" (Figura 1).

C. Tercer segmento que corresponde al canal pudendo o también conocido como de Alcock (5). Después de pasar alrededor del ligamento sacroespinoso, el NP se desplaza bajo el músculo elevador del ano, a lo largo de la tuberosidad isquiática, en una vaina de la aponeurosis del músculo obturador interno que forma el canal de Alcock (Figura 2).

La vagina, en su trayecto anatómico corre paralela a los tres segmentos del NP, lo que la transforma en una vía de abordaje lógica para acceder a la o las zonas donde existe compromiso del nervio.

\section{RECORRIDO DE LAS RAMAS Y TERMINACIO- NES NERVIOSAS DEL NERVIO PUDENDO}

La anatomía de las terminaciones nerviosas es muy compleja y tiene muchas variantes. Sin embargo, el conocimiento de las áreas sensitivas inervadas por el NP, nos va a permitir relacionar el dolor con su posible origen pudendo. ra 3):

EI NP posee tres ramas terminales $(6,7)$ (Figu-

A.- Nervio rectal inferior. En la mayoría de las pacientes comienza en el canal de Alcock. Sus ramas terminales sensitivas inervan el canal anal, el tercio caudal del recto, la piel posterior de la horquilla vulvar y perianal. Además posee terminaciones perineales cutáneas dorsales. Las terminaciones motoras alcanzan el nivel anal del elevador y el esfínter anal externo.

B.-Nervio perineal. Emerge del NP a la salida del canal de Alcock. Sus ramas sensitivas envuelven el tercio inferior de la vagina y de la uretra, y los labios mayores y menores. Las ramas motoras cruzan la membrana perineal ventral y terminan en el esfínter estriado de la uretra.

C.-Nervio dorsal del clítoris. Comienza también a la salida del canal de Alcock. Este nervio posee dos ramas, la rama clitorídea y la rama pubiana, que tienen terminaciones que van más allá de la arcada pubiana, y que alcanzan al conducto inguinal.

Además el NP inerva los músculos bulboespongioso, isquiocavernoso, elevador del ano y el transverso perineal superficial y profundo.

Las tres ramas terminales poseen en diferente proporción fibras motoras, sensitivas y autonómi- 


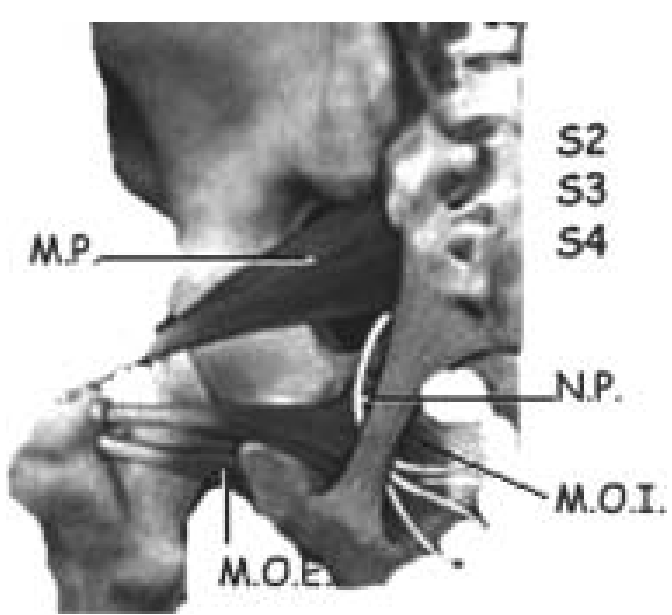

Figura 1. Vista posterior del nervio pudendo. NP: Nervio pudendo, MP: músculo piriforme, MOI: músculo obturador interno, MOE: músculo obturador externo. S2, S3, S4: Raíces sacras.

cas. Por ello su atrapamiento puede causar signos y síntomas, como veremos más adelante, de expresión en cualquiera de los tres ámbitos. En total se estima que el $30 \%$ es autonómico, y el $70 \%$ es somático ( $50 \%$ sensitivo y $20 \%$ motor) (Figura 4 ).

\section{CAUSAS DE ATRAPAMIENTO DEL NERVIO PUDENDO}

Como podemos inferir, son muy diversas:

- Profesión, oficio o práctica deportiva que implique permanecer mucho tiempo sentada. Es el caso de ciclistas, en las cuales un sillín inadecuado puede causar una compresión permanente que lleva a la larga a desencadenar un SANP (8).

- Trauma, incluso de mucho tiempo antes de la aparición de los síntomas.

- Parto vaginal y episiotomía.

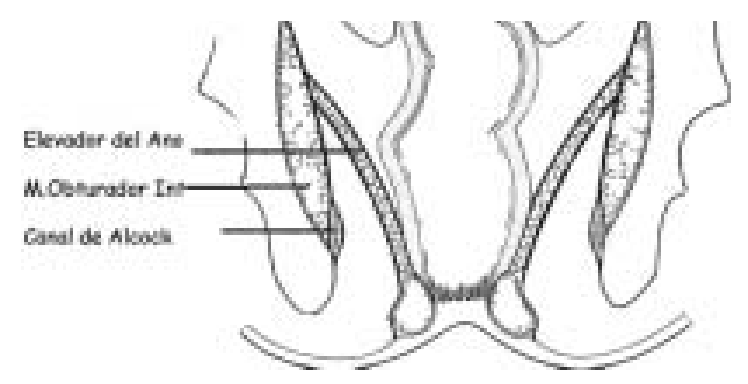

Figura 2. Corte frontal de pelvis a nivel perineal. Vista del canal de Alcock.
- Cirugía ginecológica pelviana previa, abdominal o vaginal.

- Radioterapia pelviana, que provoque cambios morfológicos y estructurales en los tejidos adyacentes, llevando a comprimir al NP.

- Defectos congénitos (óseos, musculares, etc.) que implican atrapamiento.

\section{RELACIÓN ENTRE EL SANP Y EL PARTO}

El parto vaginal, al causar distensión del músculo elevador del ano, provoca también estiramiento del NP, cuyo recorrido anatómico transcurre inmediatamente por debajo del piso pelviano. Se estima que un 30 a $40 \%$ de los partos vaginales se asocia con un grado importante de estiramiento pudendo; afortunadamente en la mayoría de los casos el nervio se recupera sin dejar secuelas. Como se puede inferir el riesgo será mayor en aquellos partos con expulsivos prolongados, partos operatorios (fórceps) y aquellos con

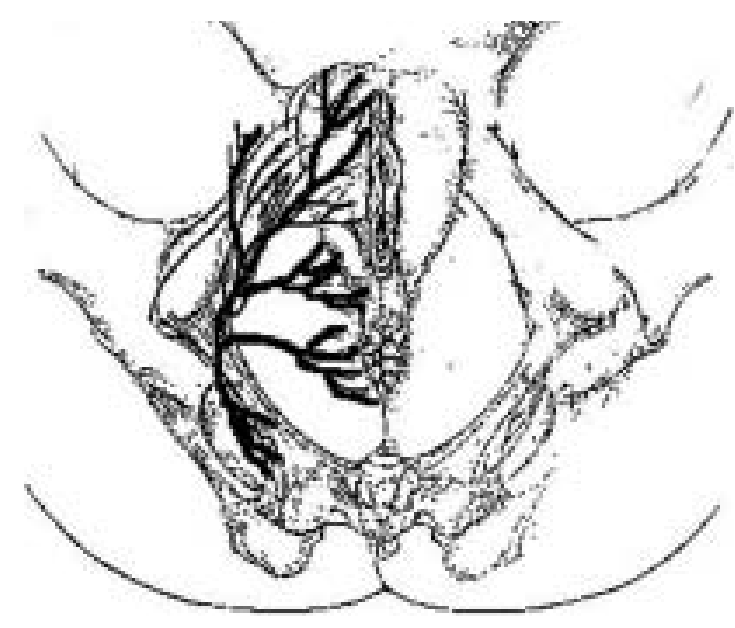

Figura 3. Vista inferior distribución anatómica del nervio pudendo.

Nervio dersal del elitoris

Nervio perined

Nervio rectal inferier

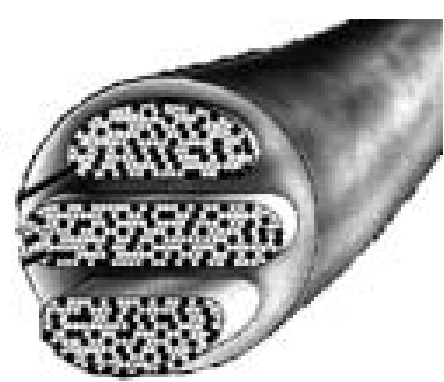

Figura 4. Esquema del nervio pudendo formado por sus tres ramas. 
fetos macrosómicos. Se estima que un descenso superior a $1,4 \mathrm{~cm}$ de la inserción inicial del piso pelviano puede ocasionar estiramiento del NP. A su vez si este estiramiento sobrepasa el $12 \%$ de la longitud inicial del nervio se genera daño neurológico definitivo. Otra posible causa asociada al parto puede ser la episiotomía lateral, amplia y descuidada, que también puede lesionar las ramas tranverso perineales de este nervio (9).

\section{DIAGNÓSTICO DEL SANP}

El dolor es la causa más común de consulta (10). Clásicamente se describe como perineal, que se agrava al sentarse, disminuye o desaparece al estar de pie, habitualmente ausente al acostarse, y no compromete el sueño. Estas pacientes relatan un dolor que aumenta al sentarse en alguna superficie plana, pero que desaparece al hacerlo en el asiento del inodoro. Además podemos ver que mientras la entrevistamos, se sienta de lado, cambiando la posición en forma reiterada, con gran incomodidad. Se trata de un dolor neuropático, que puede ser quemante, asociado a prurito, irradiado como electricidad, continuo o por descargas repentinas, y muchas veces de gran intensidad.

Este dolor puede presentarse asociado a diferentes síntomas y signos, lo cual puede dificultar aún más nuestro diagnóstico, al desviar nuestra atención a múltiples patologías de diagnóstico diferencial por compromiso de otros sistemas (gastrointestinal y urinario) (11-14). Esto explica la tardanza en realizar el diagnóstico correcto, la que se estima entre 7 a 10 años en las series publicadas.

Diferentes presentaciones del dolor en el SANP:

1. Sólo dolor.

2. Dolor con síntomas urinarios (vejiga irritable, micción con obstrucción). ción.

3. Dolor con defecación dificultosa por obstruc-

4. Dolor con disfunción sexual (dispareunia, persistente excitación).

5. Dolor con incontinencia urinaria, anal o ambas.

6. Todas las anteriores.

Un estudio de 55 pacientes con SANP presentado por Beco y cols en 2004 (15), demostró la frecuente asociación entre incontinencia anal, incontinencia urinaria y perineodinia. El 71\% (39 de 55) presentaban asociación de dos de estos signos, mientras que el 13\% (7 de 55) presentaba las tres.
Al territorio normalmente inervado por el NP, pueden asociarse áreas correspondientes a otros dermatomas (T11-12 y L1), lo que se explica por anastomosis con otros nervios (por ejemplo el femorocutáneo posterior, etc.), resultando en áreas de dolor no típico.

Signos que caracterizan dolor de origen neuropático:

- Allodinia: estímulo no doloroso que produce dolor. Por ejemplo la suave presión con una mota de algodón.

- Formicación: sensación de insectos caminando en la piel. Generalmente relatan sentir hormigas caminando.

- Parestesia: sensación anormal de quemazón, cosquilleo, hormigueo o sensación de cuerpo extraño. Muchos de esos pacientes relatan la imperiosa necesidad de retirar un cuerpo extraño que no existe.

- Cambios cutáneos: entre los signos de expresión de compromiso de fibras autonómicas podemos observar cambios en la piel de la región glútea. Es posible encontrara piel de aspecto "anserina", "marmórea" o "piel de naranja" (Figura 5).

- Inflamación: causada por neuroestimulación excesiva.

- Hiperalgesia: respuesta aumentada al estímulo doloroso.

- Hipoalgesia: respuesta disminuida al estímulo doloroso.

- Disestesia: sensación impropia producida por el estímulo.

- Metestesia: respuesta prolongada al estímulo.

\section{CRITERIOS DIAGNÓSTICOS}

Se utiliza la certificación de 2 criterios mayores o 1 criterio mayor asociado a 2 criterios menores.

A. Criterio mayor

- Área dolorosa en el trayecto del nervio o sus ramas:

- Nervio rectal inferior: ano, perineo, 1/3 inferior del recto, nalgas.

- Nervio perineal: labios menores, labios mayores, 1/3 inferior de la vagina, periuretra.

- Nervio dorsal del clítoris: área cutánea del clítoris y sínfisis pubiana

- El dolor se reproduce al comprimir el tronco pudendo (signo de Tinel) en la localización anatómica del área afectada (8).

- Infiltración exitosa en el sitio anatómico. Se produce un alivio importante o mejoría en el área afectada al inyectar lidocaina, lo que debe durar por más de 12 horas. 


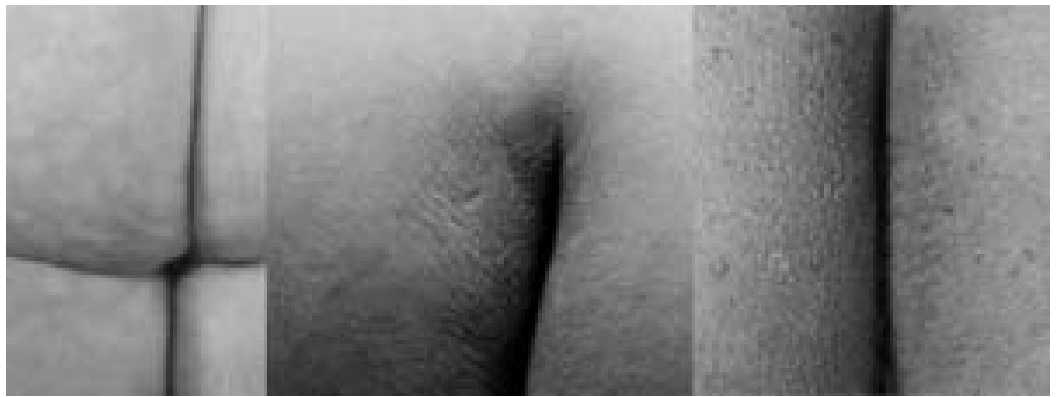

Figura 5. Efectos sobre la piel por compromiso de fibras autonómicas en el síndrome de atrapamiento del nervio pudendo. Piel "marmórea", "de naranja" y "anserina".

\section{B. Criterio menor}

- Sensación de dolor neuropático.

- Existencia de una posición dolorosa y/o antiálgica. Por ejemplo aumenta al sentarse y se alivia en decúbito.

- Existencia de un factor etiológico o un evento desencadenante. Por ejemplo, después de un parto, traumatismo, cirugía perineal, etc.

- Ausencia de otra causa que explique el dolor en el área pélvica (patología ginecológica como endometriosis, adenomiosis, etc.).

\section{PROCEDIMIENTOS DE APOYO DIAGNÓSTICO}

En general los test de compresión en los territorios inervados por el pudendo, son capaces de reproducir el dolor en la zona descrita por la paciente. Un test que se puede realizar es el llamado "Rolling Test" (15), que consiste en desplazar piel y celular subcutáneo desde el ano hacia el pubis, sobre el trayecto de las ramas del nervio pudendo (Figura 6). Al realizar esta maniobra, se reproducirá el dolor en la zona inervada. Igualmente efectivo resulta desencadenar dolor provocando compresión del nervio en puntos determinados, como la espina isquiática o el canal de Alcock.

\section{EXÁMENES DE APOYO DIAGNÓSTICO}

- Estudio electrofisiológico (16): La electromiografía (EMG) de los músculos inervados por el nervio pudendo. La EMG radicular permite confirmar el daño a nivel medular o en el origen de las raíces sacras. La EMG de los músculos inervados por el NP y el reflejo sacro, permiten localizar el área de compromiso anatómico. Además se utilizan test de conducción nerviosa, entre los cuales se encuentran el Pudendal Nerve Distal Motor Latency Test (PNDMLT), cuyo valor normal superior es 2,2 mseg (endorectal) y los test sensitivos, siendo los dos principales, el Quantitative Sensitive Treshold (QST) y el Warm Treshold Detection
(WTD). De este modo es posible localizar el área de compromiso anatómico, por ejemplo, compresión del nervio por la pinza interligamentos, o en el canal de Alcock. El estudio electrofisiológico confirma el daño pudendo en la mitad de los casos; se piensa que el realizar el examen en una posición diferente a la que causa dolor explicaría su resultado falsamente negativo.

- Imageneología: El método más utilizado por la gran resolución que entrega en el examen de la región lumbosacra y el plexo sacro.

- Evaluación fisioterapéutica: Con lo cual se registra o descarta el aumento del tono muscular del piso pelviano, que en la mayoría de las pacientes se encuentra elevado por una reacción de tipo reflejo muscular.

\section{LA IMPORTANCIA DE LA CLÍNICA EN EL DIAGNÓSTICO}

La historia clínica del paciente es fundamental en el diagnóstico (17). Por lo tanto se deben utilizar preguntas predeterminadas para ello. Si los estudios de laboratorio, como la electromiografía,

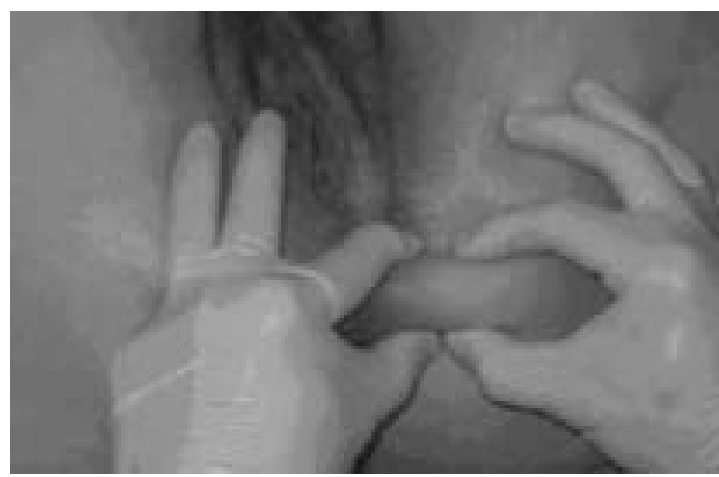

Figura 6. Rolling Test. Desplazamiento de la piel y celular subcutáneo desde el ano hacia el pubis, sobre el trayecto de las ramas del nervio pudendo. Al realizar esta maniobra, se reproducirá el dolor en la zona inervada. 
no concuerdan con los hallazgos clínicos, el diagnóstico se debe apoyar en estos últimos (18).

Cuestionario de SANP (19):

- ¿Cuánto tiempo que tiene dolor?

- ¿Hay algún evento que coincidió con el inicio del dolor?

- ¿Cuánto duele de 0 a 10 ?

- ¿El dolor es: "quemante", "tirante", "aplastante", "presión", "penetrante", "cortante", otros (señalar con un círculo sobre el tipo de dolor)?

- ¿El dolor es mayor en un lado de mis "asentaderas" que en el otro.

- ¿Tengo dolor en la región de las nalgas?

- ¿Tengo dolor en la vagina?

- ¿Tengo dolor en los labios de la vulva?

- ¿El dolor es peor al sentarse?

- ¿Sentarse en el baño no es tan doloroso como sentarse en una superficie plana?

- ¿El dolor es peor al estar de pie?

- ¿El dolor es peor al acostarse?

- ¿El dolor es peor al acostarse hacia un lado que al otro?

- ¿Hay dolor con la defecación?

- ¿Hay dolor con la relación sexual?

- ¿Hay dolor al orinar?

- ¿Tengo problemas para controlar mi vejiga? no?

- ¿Tengo problemas para controlar mi intesti-

- ¿Tengo problemas para controlar la expulsión de gases?

En cada una conteste Sí o NO

\section{TRATAMIENTO}

\section{A. Tratamiento médico}

Se han utilizado diversos medicamentos con diferentes grados de respuesta y efectividad. Entre ellos la aminotriptilina, antiepilépticos (como la gabapentina), lidocaína en gel o parches, narcóticos, etc. Siempre es recomendable asociarlos al resto de la terapia. El mejor esquema de tratamiento es secuencial y comprende las siguientes etapas:

Etapa 1: Autocuidado (hiperprotección perineal). Dejar de hacer actividades que causan o gatillan el dolor (sentarse, ciclismo, etc). Uso de plataforma perineal, cuyo propósito es recibir el peso corporal sobre las tuberosidades isquiáticas al sentarse, dejando el periné libre de compresión.

Etapa 2: Inyecciones perineurales (PNPI): corticoides y lidocaína/bupivacaína, 2 en el espacio interligamentoso y 1 en el canal de Alcock , bajo fluoroscopía o CT scan. Esta técnica produce dramática definición de los síntomas al aliviarlos: alivian el dolor neuropático, liberan la obstrucción de esfínteres, suprimen síntomas vesicales, normalizan la disfunción sexual. Durables desde horas, semanas, máximos hasta $>2$ años, algunos otorgan curación; dependiendo del uso solo o combinado de anestésicos y corticoides. El alivio sintomático depende de la exactitud de la ubicación (anatomía altamente variable). La respuesta sensitiva, motora o autonómica es variable, lo que la hace impredecible. Se administran cada 4 a 6 semanas y con un máximo de 6 infiltraciones, midiendo la respuesta cutánea post PNPI y monitorizando los resultados cada semana. Si no hay respuesta adecuada se procede a la tercera etapa.

\section{B. Tratamiento quirúrgico}

Etapa 3: Este tratamiento tiene por objetivo lograr la descompresión del nervio pudendo. Entre los hallazgos que podemos encontrar en la cirugía destacan:

- Ligamentos hipertróficos a menudo con bordes agudos y prominencias.

- Ligamentos fusionados (funicular).

- Bandas ligamentosas que atraviesan el nervio.

- Adherencias.

- Unión y compresión contra el ligamento sacrotuberoso.

- Ramificación proximal: rama rectal inferior que penetra el ligamento sacroespinal.

- Tronco nervioso principal atraviesa el músculo coccígeo.

- Espina isquiática elongada.

El atrapamiento del NP habitualmente ocurre:

- Entre el Ligamento Sacroespinoso y el Ligamento Sacrotuberoso (pinza interligamentosa, 70\% de los casos).

- En el canal de Alcock, 20\%.

- En el diafragma urogenital (Nervio Dorsal del Clítoris).

- En $20 \%$ de los casos existe compromiso simultáneo interligamentoso y canal de Alcock.

Las diferentes vías de abordaje pueden ser:

- Técnica transperineal (20)

- Técnica transglútea (8)

- Técnica laparoscópica (21)

- Técnica transvaginal (22).

Se accede a los tres segmentos del NP a través de la fosa isquirectal, con la paciente en posición de litotomía y a través de la vagina por la cual se alcanza este espacio avascular. Su disec- 
ción permite exponer la cara medial de cada lado de la pelvis después de rechazar el recto y el elevador del ano. El propósito es la descompresión de los lugares comprometidos, por lo tanto no hay neurolisis ni disección cercana al nervio, lo que evita desvascularización o adelgazamiento del nervio que ya tiene obvios daños.

Las técnicas quirúrgicas iniciales sugerían la necesidad de seccionar los ligamentos sacroespinoso y sacrotuberoso y la transposición del NP (23) por delante de la espina ciática. Sin embargo, se ha visto que la descompresión bien realizada no hace necesaria la movilización del NP, obteniéndose iguales resultados. La comparación de las técnicas se aprecia en la Tabla I.

Durante el post-operatorio en un $38 \%$ de los casos se produce un dolor intenso en la región glútea, diferente al de ingreso, que puede atribuirse a la sección del ligamento sacroespinoso y la abundancia de la red nerviosa de algunos de ellos. Disminuye progresivamente el primer mes y termina desapareciendo por completo.

La descompresión comienza siempre por abrir el canal bajo el músculo piriforme, en el borde inferior del ligamento sacroespinoso. Una vez que se alcanza el tejido adiposo perineural, se procede a explorar la zona de la compresión. En 90 \%, la sección del ligamento sacro espinoso desde superior a inferior se lleva a cabo para liberar la pinza.

La operación puede proseguir hacia caudal, si es necesario, cortando las fibras del proceso falciforme del ligamento sacrotuberoso hasta penetrar en el canal de Alcock. En esta etapa, se explora digitalmente todo el canal completando la descom- presión y asegurándose que todo el canal pudendo está libre. Esta aproximación parece la ideal por 3 razones:

- Es la única técnica que permite acceso a los 3 segmentos del NP y en particular a la zona de los ligamentos, asiento de la mayoría de las compresiones.

- La descompresión compromete exclusivamente aquellas fibras patológicas de intensa fibrosis que aprisionan el NP contra el ligamento ósteopélvico.

- No se tocan otras estructuras.

La formación de hematomas se previene dejando en forma rutinaria con la colocación de un drenaje en la región isquiorectal por 48 horas. Se utiliza sonda Foley durante la cirugía y se retira 24 horas después.

Alta al $3^{\text {er }}$ ó $4^{\circ}$ día. Luego kinesioterapia antálgica y de reeducación perineal.

La cirugía termina con la colocación de un catéter para infusión analgésica, en el canal de Alcock abierto, hasta el nivel subpubiano. Se retira el $3^{\text {er }}$ ó $4^{\circ}$ día. En aquellos casos en que se sospeche la posibilidad de un dolor de largo plazo el catéter se conecta a una cámara que se implanta in situ; evita las descargas neurálgicas durante el proceso de regeneración nerviosa que toma de 6 a 18 meses.

La evaluación del resultado quirúrgico debe ser realizada a corto y largo plazo, ya que la recuperación de un nervio dañado puede tomar tiempos largos, habitualmente superiores a un año. Las primeras series publicadas de descompresión quirúrgica comprenden grupos reducidos de pacientes; en la medida que se ha reconocido el

Tabla I

COMPARACIÓN DE LAS VÍAS DE ABORDAJE QUIRÚRGICO EN LA DESCOMPRESIÓN DEL NERVIO PUDENDO

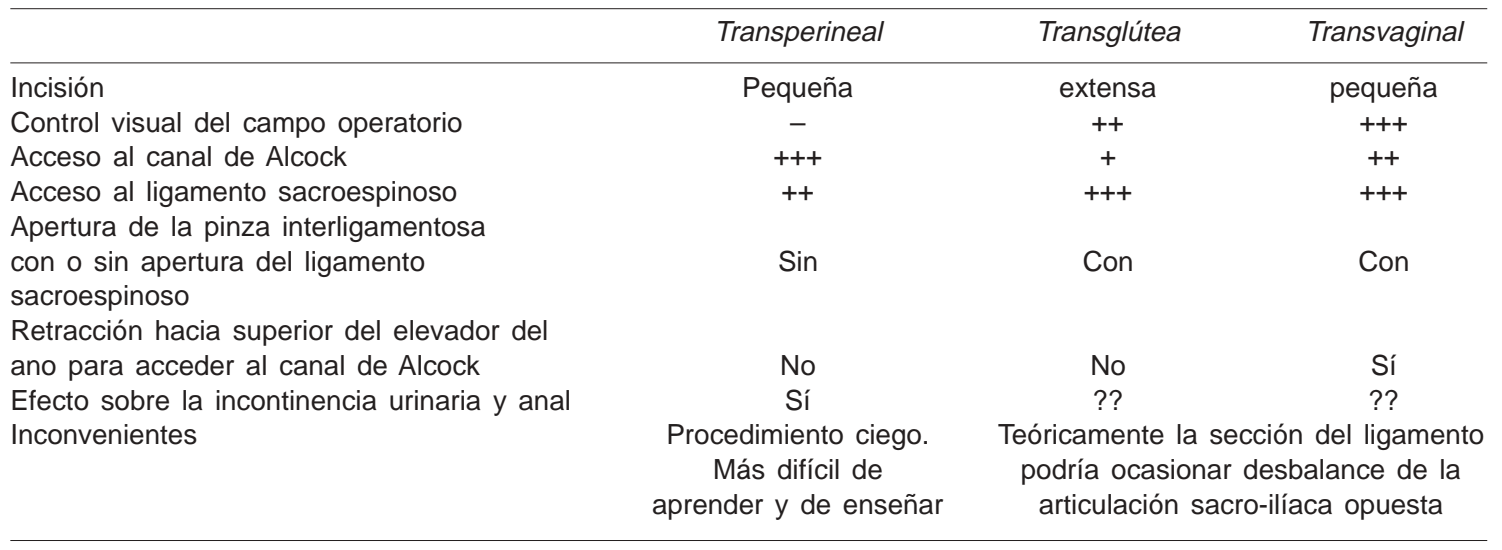


Tabla II

PUBLICACIONES DE CIRUGÍAS DE DESCOMPRESIÓN DE NERVIO PERINEAL

\begin{tabular}{llcccccc}
\hline Autor & Tipo de trabajo & Año & $\begin{array}{c}\text { Número de } \\
\text { pacientes }\end{array}$ & $\begin{array}{c}\text { Seguimiento } \\
\text { máximo }\end{array}$ & $\begin{array}{c}\text { Mejoría } \\
\text { Mejoría } \\
\text { parcial }\end{array}$ & $\begin{array}{c}\text { Sin } \\
\text { cambios }\end{array}$ \\
\hline Bascom(28) & Prospectivo descriptivo & 1988 & 7 & 19 meses & $45 \%$ & $33 \%$ & $22 \%$ \\
Bautrant(22) & Prospectivo descriptivo & 2003 & 104 & 1 año & $62 \%$ & $23 \%$ & $15 \%$ \\
Beco (15) & Retrospectivo comparativo & 2004 & 74 & 9 años & $61,10 \%$ & $13,60 \%$ & $22,3 \%$ \\
Robert (24) & Controlado randomizado & 2005 & 25 & 4 años & $71,40 \%$ & $12,60 \%$ & $16,00 \%$ \\
Benson(19) & Descriptivo retrospectivo & 2005 & 10 & 2 años & $60 \%$ & - & - \\
\hline
\end{tabular}

diagnóstico, el número de pacientes sometidas a descompresión quirúrgica ha ido progresivamente en aumento y autores como Robert (24) han reportado más de 400 cirugías desde 1989, Bautrant (22) reporta 104 descompresiones y Ansell (18) 170. Los resultados se analizan en la Tabla II.

Las complicaciones descritas en la cirugía de descompresión son menores (18):

- Infecciosas: ITU (7\%), abscesos (2,5\%) con evacuación y drenaje.

- Hemorrágicas: $2 \%$, injuria a rama de la arteria pudenda y falso aneurisma secundario en rama de la arteria glútea cranial.

La teórica desestabilización y sobrecarga de la articulación sacroilíaca como complicación de la sección de los ligamentos no han sido demostradas hasta ahora en las publicaciones conocidas.

\section{COMPROMISO NO NEUROPÁTICO DEL NERVIO PUDENDO (25-27)}

Nunca debe considerarse el compromiso pudendo como neuropático hasta haber descartado totalmente la posibilidad de patología orgánica, como endometriosis, metástasis o várices pelvianas, que son capaces de producir compresión o invasión del canal de Alcock.

\section{CONCLUSIONES}

El atrapamiento del NP constituye causa de dolor pelviano crónico en la mujer, siendo uno de los diagnósticos menos conocidos y subestimados hasta hoy. El conocimiento de este síndrome, que afectaría hasta en un $15 \%$ a las mujeres en alguna etapa de su vida, permitirá establecer su verdadera prevalencia e incidencia en la población femenina. La anamnesis y el examen físico dirigido son las mejores herramientas para un correcto diagnóstico, que puede ser complementado con estudios específicos de elecrofisiología. El apoyo de imágenes básicamente nos ayuda a descartar patología orgánica. El esquema secuencial de tratamiento es la opción más adecuada para cada caso y la cirugía de descompresión queda reservada para aquellos casos que no responden a terapias conservadoras. Los mejores resultados de la cirugía se harán evidentes mientras más precoz se haga el diagnóstico, ya que la recuperación neurológica de un nervio dañado por largo tiempo puede ser muy lenta, incompleta o incluso ausente. De manera que sólo la sospecha diagnóstica precoz permitirá un real impacto en la mala calidad de vida que sufre una mujer aquejada de esta patología.

\section{BIBLIOGRAFÍA}

1. Amarenco G, Lanoe $Y$, Perrigot M, Goudal H. A new canal syndrome: compression of the pudendal nerve in Alcock's canal or perinal paralysis of cyclists. Presse Med 1987;16(8):399.

2. Robert R, Labat JJ, Lehur PA, Glemain P, Armstrong O, Le Borgne J, Barbin JY. Clinical, neurophysiologic and therapeutic remarks from anatomic data on the pudendal nerve in some cases of perineal pain. Chirurgie 1989;115(8):515-20.

3. Amarenco G, Kerdraon J, Bouju P, Le Budet C, Cocquen AL, Bosc S, Golldet R. Treatments of perineal neuralgia caused by involvement of the pudendal nerve. Rev Neurol (Paris) 1997;153(3):331-4.

4. Shafik A. Pudendal canal syndrome: a cause of chronic pelvic pain. Urology 2002;60(1):199.

5. Shafik A, Doss SH. Pudendal canal: surgical anatomy and clinical implications. Am Surg 1999;65(2): 176-80.

6. Shafik A, EL-Sherif M, Youssef A, Olfat ES. Surgical anatomy of the pudendal nerve and its clinical implications. Clin Anat 1995;8(2):110-115.

7. Hollabaugh RS Jr, Steiner MS, Sellers KD, Samm BJ, Dmochowski RR. Neuroanatomy of the pelvis: implications for colonic and rectal resection. Dis Colon Rectum 2000;43(10):1390-7.

8. Amarenco G, Lanoe Y, Ghnassia RT, Goudal H, 
Perrigot M. Alcock's canal syndrome and perineal neuralgia. Rev Neurol (Paris) 1988;144(8-9):523-6.

9. Lien KC, Morgan DM, DeLancey JO, Ashton-Miller JA. Pudendal nerve stretch during vaginal birth: a 3D computer simulation. Am J Obstet Gynecol 2005; 192(5):1669-76.

10. Robert R, Brunet C, Faure A, Lehur PA, Labat JJ, Bensignor M, Leborgne J, Barbin JY. Surgery of pudendal nerve in various types of perineal pain: course and results. Chirurgie 1993-94;119(9):535-9.

11. Shafik A. Pudendal canal descompression for the treatment of fecal incontinence in complete rectal prolapse. Am Surg 1996;62(5):339-43.

12. Shafik A. Role of pudendal canal syndrome in the etiology of fecal incontinence in rectal prolapse. Digestion 1997;58(5):489-93.

13. Shafik A. Pudendal canal syndrome and proctalgia fugax. Dis Colon Rectum 1997;40(4):504.

14. Shafik A. Pudendal canal syndrome as a cause of vulvodynia and its treatment by pudendal nerve descompression. Eur J Obstet Gynecol Reprod Biol 1998;80(2):215-20.

15. Beco J, Climov D, Bex M. Pudendal nerve decompression in perineology: a case series. BMC Surg 2004;30(4):15. Hallado en:

http://www.biomedcentral.com/1471-2482/4/15

16. Galaup JP. Dyschezia, pudendal neuropathy, and functional pelvico-perineal disorders. Prog Urol 2000; 10(1):114-22.

17. Turner ML, Marinoff SC. Pudendal neuralgia. Am J Obstet Gynecol 1991;165(4 Pt 2):1233-6.

18. Antolak SJ Jr, Hough DM, Pawlina W, Spinner RJ. Anatomical basis of chronic pelvic pain syndrome: the ischial spine and pudendal nerve entrapment. Med Hypotheses 2002;59(3):349-53.

19. Benson JT, Griffis K. Pudendal neuralgia, a severe pain syndrome. Am J Obstet Gynecol 2005;192(5): 1663-8.

20. Shafik A. Pudendal canal decompression in the treatment of erectile dysfunction. Arch Androl 1994 32(2):141-9.

21. Shafik A. Endoscopic pudendal canal decompression for the treatment of fecal incontinence due to pudendal canal syndrome. J Laparoendoscopic Adv Surg Tech A 1997;7(4):227-34.

22. Bautrant E, de Bisschop E, Vaini-Elies V, Massonnat $\mathrm{J}$, Aleman I, Buntinx $\mathrm{J}$, et al. La prise en charge moderne des névralgies pudendales. A partir d'une série de 212 patientes et 104 interventions de décompression. J Gynecol Obstet Biol Reprod (Paris) 2003;32:705-12.

23. Mauillon J, Thoumas D, Leroi AM, Freger P, Michot F, Denis $P$ Results of pudendal nerve neurolysistransposition in twelve patients suffering from pudendal neuralgia. Dis Colon Rectum 1999;42(2):186-92.

24. Robert R, Labat JJ, Bensignor $M$, Glemain $P$, Deschamps C, Raoul S, et al. Decompression and transposition of the pudendal nerve in pudendal neuralgia: a randomized controlled trial and long-term evaluation. Eur Urol 2005;47(3):403-8.

25. Nehme-Shuster $\mathrm{H}$, Youssef $\mathrm{C}$, Roy C, Brettes JP, Martin T, Pasquali JL, et al. Alcock's canal syndrome revealing endometriosis. Lancet 2005;366(9492): 1238.

26. Moser T, Scheiber-Nogueira MC, Nogueira TS, Doll A, Jahn C, Beaujeux R. Neurological picture. Pudendal nerve compression by pelvic varices: successful treatment with transcatheter ovarian vein embolisation. J Neurol Neurosurg Psychiatry 2006;77(1):88. doi:10.1136/jnnp.2005.069278.

27. Thomas E, Abiad L, Cyteval C, Gallix B, Taourel P, Bruel JM, et al. Metastic pudendal nerve compression presenting as atypical sciatica. J Spinal Disord Tech 2002;15(4):324-5.

28. Bascom JU. Pudendal canal syndrome and proctalgia fugax: a mechanism creating pain. Dis Colon Rectum 1998;41(3):406. Letter. 